\title{
Probing nuclear matter with jet conversions
}

\author{
W. Liu ${ }^{1}$ and R. J. Fries ${ }^{1,2}$ \\ ${ }^{1}$ Cyclotron Institute and Department of Physics, Texas A\&M University, College Station, Texas 77843, USA \\ ${ }^{2}$ RIKEN/BNL Research Center, Brookhaven National Laboratory, Upton, New York 11973, USA
}

(Received 31 January 2008; published 22 May 2008)

\begin{abstract}
We discuss the flavor of leading jet partons as a valuable probe of nuclear matter. We point out that the coupling of jets to nuclear matter naturally leads to an alteration of jet chemistry even at high transverse momentum $p_{T}$. In particular, quantum chromodynamics $(\mathrm{QCD})$ jets coupling to a chemically equilibrated quark gluon plasma in nuclear collisions will lead to hadron ratios at high transverse momentum $p_{T}$ that can differ significantly from their counterparts in $p+p$ collisions. Flavor measurements could complement energy loss as a way to study interactions of hard QCD jets with nuclear matter. Roughly speaking they probe the inverse mean free path $1 / \lambda$ while energy loss probes the average squared momentum transfer $\mu^{2} / \lambda$. We present some estimates for the rate of jet conversions in a consistent Fokker-Planck framework and their impact on future high- $p_{T}$ identified hadron measurements at RHIC and LHC. We also suggest some novel observables to test flavor effects.
\end{abstract}

DOI: 10.1103/PhysRevC.77.054902

PACS number(s): 24.85.+p, 12.38.Mh, 21.65.-f, 25.75.-q

\section{INTRODUCTION}

A hot and dense state of matter, called quark gluon plasma (QGP), is believed to have formed during the first few microseconds after the big bang. Its degrees of freedom above a critical temperature $T_{c}$ consist of deconfined quarks and gluons. The Relativistic Heavy Ion Collider (RHIC) was built to study the formation and properties of quark gluon plasma in collisions of heavy ions at very large energies. We have overwhelming evidence from the first few years of running of RHIC that the temperatures reached are well above $T_{c}$ and that the new matter is indeed partonic [1].

One of the key observations at RHIC is the suppression of hadrons with large transverse momentum $p_{T}[2,3]$, most impressively manifested in reduced nuclear modification factors $R_{A A} \approx 0.2$ for hadrons in the most central collisions for $p_{T}>5 \mathrm{GeV} / c . R_{A A}$ measures the ratio of yields in nuclear collisions $(A+A)$ vs nucleon-nucleon collisions $(N+N)$ at the same energy scaled with the appropriate number of binary collisions. High- $p_{T}$ hadrons above $5 \mathrm{GeV} / c$ in $A+A$ collisions are believed to come predominantly from fragmentation off quantum chromodynamics (QCD) jets. QCD jets originate from high- $p_{T}$ partons created in the initial hard collisions between the nucleons in the nuclei. The jet quenching phenomenon at RHIC has been attributed to loss of energy of the high- $p_{T}$ partons on their way through the medium [4-9]. It is often characterized by a transport coefficient $\hat{q}=\mu^{2} / \lambda$, a ratio of the average squared momentum transfer $\mu^{2}$ per collision and the mean free path $\lambda$.

Initially, energy loss had been attributed to induced gluon radiation by scattering of the leading jet parton with thermal partons from the medium. Perturbative calculations of this process yield results for $R_{A A}$ compatible with data after fixing one parameter characterizing the strength of the interaction, the transport coefficient $\hat{q}$ or an equivalent quantity. However, recently several inconsistencies have led to considerable efforts to revisit this topic. First, the values for the transport coefficients extracted from measurements of $R_{A A}$ have large uncertainties and differ between the various theoretical models [10]. Second, the description of observables beyond those based on the simple single inclusive spectrum is far more problematic. As an example we mention the azimuthal anisotropy $v_{2}$ at high $p_{T}$ that is larger than that predicted by theory [11]. Third, the application of radiative, perturbative energy loss to heavy quarks leads to the prediction of much smaller quenching than for light quarks, in contradiction with data from RHIC [12].

The partial failure of radiative, perturbative energy loss led to renewed interest in energy loss from elastic collisions [13-15], although a self-consistent treatment of elastic and radiative energy loss in one proper unified theoretical framework is still under construction. It has also ushered in a period of renewed interest in nonperturbative mechanisms. Examples are energy loss through bound states in the plasma [16] — successfully applied to heavy quarks — and energy loss in the infinite coupling limit of QCD, modeled through the anti-de-Sitter space/conformal field theory (AdS/CFT) correspondence [17].

In this work, we discuss the potential of flavor-dependent measurements of jet quenching observables. It is obvious that the interaction of a jet with the medium can change the flavor of the jet, defined here as the flavor of the leading parton. We will show that this leads to observable differences in the final jet hadron chemistry. Our generalized definition of flavor here includes light quarks $q(u, d, s)$, gluons $g$, and photons $\gamma$. Heavy flavors $Q(c$ and $b$ ) should also be included. There is no doubt that flavor-changing processes exist for fast partons coupling to a medium. Indeed, flavor-changing channels have been included in computations of medium-modified fragmentation functions [18], and quark-gluon conversions of the leading jet particle are also implemented in the ArnoldMoore-Yaffe (AMY) formalism [9]. Nevertheless, flavorchanging processes are often neglected in phenomenological studies.

This situation led to the widespread opinion that quark and gluon jets are well-defined concepts in a medium and that they ought to exhibit a relative suppression by a color 
factor $9 / 4$. This claim might have some validity in the case that the mean-free path of the leading particle is of the order of (or larger than) the size of the medium, $\lambda \approx L$. Energy loss is then dominated by emission of only one gluon. In general, however, the situation is different. We want to emphasize that the flavor of a leading parton might be well-defined only locally and that it can change along the trajectory. Recently, Sapeta and Wiedemann argued that changes in jet chemistry could also arise from the increased multiplicity in medium-modified jets [19]. In a model using enhanced parton branching they find effects similar to those discussed by us here.

Nevertheless, most studies of jets in nuclear matter still focus solely on the kinematical effects, based on quenching of longitudinal momentum and broadening of transverse momentum of the leading parton. For example, observables like $R_{A A}$ and $v_{2}$ are sensitive to various integrals over the differential energy loss $d E / d x$ and ultimately measure the momentum transfer per path length, i.e., $\hat{q}$. We can summarize this as the effect of jets coupling to the thermal properties of the medium. We like to advocate a second, complementary approach that looks at flavor effects from jets coupling to the chemical properties of the medium. Measurements of identified hadrons at high- $p_{T}$ can constrain the rate of conversions. With sufficient experimental sensitivity this would lead to estimates for the mean free path $\lambda$ of the jet in the medium, complementary to measurements of $\hat{q}$. We expect such measurements to provide additional stringent tests for the validity of any model for the jet-medium coupling. Of course, with any novel observation different explanations have to be taken into account.

This work is organized as follows. In the next section we discuss all relevant conversion channels on a qualitative level and assess their relative importance. We focus on jets interacting with a deconfined quark gluon plasma in nuclear collisions, but our arguments can be easily applied to cold or hot hadronic matter as well. We then proceed to present numerical results for RHIC and LHC in a consistent model based on rate equations for conversions and a FokkerPlanck equation for energy loss, using perturbative leadingorder (LO) cross sections. The formalism is introduced in Sec. III while a discussion of the results can be found in Secs. IV and V. We focus on light and strange quarks and real photons. Conversions including heavy quarks will not affect the abundance of light species and photons measurably. Therefore, the effect on charm and bottom spectra will be addressed in a separate publication [20]. We will also introduce some novel double-ratio observables that might be particularly sensitive to conversion processes. Finally, a summary and discussion are presented in Sec. VI.

\section{FLAVOR CONVERSIONS IN QUARK GLUON PLASMA}

Let us start by discussing possible flavor conversion processes in a leading order perturbative approach. Annihilation and pair creation processes (i) $q+\bar{q} \leftrightarrow g+g$ can lead to a conversion of quarks into gluons and vice versa. In the following we agree that the first parton mentioned on either side of an arrow $\leftrightarrow$ or $\rightarrow$ is the leading jet parton in the sense that it is the one with the larger momentum with respect to the local rest frame of the medium among all other partons on the same side of the arrow. $q$ can be a quark or antiquark and $\bar{q}$ is its antiparticle.

Photons can be easily created through (ii) $q+\bar{q} \rightarrow \gamma+g$ and (iii) $q+\bar{q} \rightarrow \gamma+\gamma$. Note that we can neglect the opposite processes and generally all processes with photons in the initial state for obvious reasons. It is also safe to ignore $q+\bar{q} \rightarrow g+\gamma$, which in our notation contributes to the yield of gluon jets but is much suppressed compared to the annihilation contribution (i). However, flavor changing Compton processes (iv) $q+g \leftrightarrow g+q$ are as important as annihilation and pair creation. Compton processes also contribute to photon production via (v) $q+g \rightarrow \gamma+q$.

Let us comment on the fate of heavy quarks. Heavy quark annihilation is negligible at realistic temperatures, but the opposite process (vi) $g+g \rightarrow Q+\bar{Q}$ might be an important source of excess heavy quarks. The Compton process for heavy quarks (vii) $Q+g \rightarrow g+Q$ is interesting as well because it can effectively accelerate heavy quarks from the medium or decelerate existing fast heavy quarks. We will report on the quantitative effects of processes involving heavy quarks in a forthcoming publication [20].

Some of the conversion channels (i) to (v) have been investigated in the past. In Ref. [21] the authors discussed the conversion of quark into gluon jets and vice versa through annihilation and Compton channels (i) and (iv), respectively. In the absence of flavor conversions the relative suppression of gluon relative to quark jets is expected to be 9/4. Conversions reduce the difference between the nuclear modification factors for high- $p_{T}$ quarks and gluons. Assuming gluon dominated proton fragmentation functions, as given in modern parametrizations [22], this leads to an enhancement of the $p / \pi^{+}$and $\bar{p} / \pi^{-}$ratios at high transverse momentum. This is supported by recent data from the STAR Collaboration at RHIC $[23,24]$ which shows rather large $p / \pi^{+}$ratios in $\mathrm{Au}+\mathrm{Au}$ at high $p_{T}$. Models without conversion predict consistently lower $p / \pi^{+}$and $\bar{p} / \pi^{-}$ratios [25].

Interestingly, in Ref. [21] the required conversion rates were found to be much larger than those given by the leading-order perturbative calculation. An enhancement factor $(K \approx 4$ ) was necessary to describe the data. Although there are some uncertainties from the fragmentation functions this either points to large higher-order corrections [26] or to a nonperturbative mechanism favored in a strongly coupled QGP scenario [1].

It was first pointed out in Ref. [27] that annihilation and Compton processes can also lead to the conversion of a quark or gluon jet into a photon. Perturbative estimates of this process at leading order indicate a sizable contribution to the total direct photon yield at intermediate $p_{T}$ between 3 and $6 \mathrm{GeV} / c$ at RHIC energies [28-31]. The same was found for the production of virtual photons and lepton pairs $[31,32]$. Measurements of single inclusive direct photon production from the PHENIX experiment [33] are not conclusive so far. The data have been described equally well within error bars both by calculations taking into account only prompt hard photons and thermal photons and by calculations adding jet-photon conversions as well [34]. 
Recently, it was also noticed that jet-photon conversions lead to photons with an azimuthal asymmetry $v_{2}$ that is negative with respect to the $v_{2}$ of hadrons and other photon sources [35]. In Ref. [35] it was estimated that the sum of all direct photon sources leads to small and negative direct photon $v_{2}$ between 3 and $5 \%$ at intermediate $p_{T}$. However, later it was noted that the result depends crucially on details of the fireball simulation and might be much closer to zero [31]. First experimental results are compatible with zero with sizable uncertainties [36,37].

The current experimental situation can be summarized by noting that both for conversions into photons and for quarkgluon conversions no final conclusion has been reached about a direct observation of these channels. This should change in the future with extended hadron identification capabilities at RHIC and increased statistics for direct photons. Of course, the experimental confirmation of one conversion signal would be a strong indication that conversion in general, as advocated here, is an important process.

In the remainder of the article we present a calculation of conversion channels (i), (ii), (vi), and (v) in one consistent framework. We are going to compute nuclear modification factors $R_{A A}$ for pions, kaons, and direct photons and the elliptic flow $v_{2}$ of direct photons. This leads to a direct quantitative connection between the conversions into photons [27] and conversions into gluons [21].

Furthermore, we suggest the relative yield of strange hadrons at high $p_{T}$ as a new signature for jet medium coupling. The ratio of $s$ quarks to the sum of $u$ and $d$ quarks,

$$
w=\frac{s}{u+d},
$$

is about $5 \%$ for the initial leading jet particle at RHIC energies at a typical $p_{T}$ of about $10 \mathrm{GeV} / c$. It can be easily traced back to the small fraction of strange quarks in the incident nucleons. A rough estimate with CTEQ5M parton distributions [38], assuming dominance of Compton channels in the initial hard scattering, would give

$$
\begin{aligned}
w_{\text {jet }}\left(p_{T}\right. & =10 \mathrm{GeV} / c) \approx w_{\text {pdf }}(x \approx 0.1, Q \approx 10 \mathrm{GeV}) \\
& \approx \frac{0.07}{1.1} \approx 6.4 \%,
\end{aligned}
$$

consistent with the result from the full calculation. However, in a chemically equilibrated quark gluon plasma at a given temperature $T$ the ratio is

$$
w_{\mathrm{ce}}(T) \approx \frac{m_{s}^{2}}{4 T^{2}} K_{2}\left(m_{s} / T\right) .
$$

assuming massless $u$ and $d$ quarks. For a strange quark mass of $m_{s}=100 \mathrm{MeV}$ the ratio is almost $1 / 2$ even at $T_{c} \approx$ $180 \mathrm{MeV}: w_{\mathrm{ce}}\left(T_{c}\right)=0.47$. The difference between $w_{\text {jet }}$ and $w_{\text {ce }}$ is rather large and we have to expect that the relative abundance of strange quark jets will rise with time.

For an infinite medium the particle ratios of the jets would equilibrate to that of the medium (the same will happen to their momentum distribution, making them indistinguishable from the medium). For a finite path length $L$, the rate of equilibration should be a good measure of the strength of the coupling to the medium. In particular, it should give a good estimate of the mean free path $\lambda$ between flavor changing scatterings. The approach to equilibrium will be determined by the ratio $\lambda / L$.

The final parton abundances, once a jet leaves the medium, will translate into hadron abundances as given by the fragmentation process. Our poor knowledge of fragmentation functions limits our ability to make precise predictions for absolute hadron ratios. Hence, we propose to use double ratios of abundances measured in $A+A$ and $p+p$ collisions. The first ratio we are going to look at is that for direct photons relative to pions

$$
r_{\gamma / \pi^{+}}=\frac{\left(\gamma / \pi^{+}\right)_{A A}}{\left(\gamma / \pi^{+}\right)_{p p}} .
$$

Note that formally this is the same as $R_{A A}^{\gamma} / R_{A A}^{\pi}$. In such double ratios uncertainties from the determination of the number of $N+N$ collisions (needed for $R_{A A}$ ) cancel. On the theoretical side the large uncertainties from fragmentation functions tend to cancel between the $A+A$ and the $p+p$ contribution. On the experimental side, we would hope that the systematic errors for a direct extraction of double ratios are smaller than for an a posteriori reconstruction from single ratios. We will also take a fresh look at the relative abundances of protons and pions

$$
r_{p / \pi^{+}}=\frac{\left(p / \pi^{+}\right)_{A A}}{\left(p / \pi^{+}\right)_{p p}} .
$$

Generally, in $A+A$ collisions we expect the relative abundances of flavors to tend toward equilibrium values. Therefore, we expect an increase of photons compared to quark and gluons and we expect an increase of gluons relative to quarks [21]. Under the assumption that protons are dominated by fragmentation from gluons we expect the $p / \pi^{+}$ratio to increase in $A+A$ collisions, whereas $\gamma / \pi^{+}$will show an even steeper increase. A similar increase should also be observed in the relative abundance of strange hadrons as high- $p_{T}$ strange quarks tend toward equilibrium. In the remainder of the article we will try to verify our claims numerically.

\section{JET PROPAGATION IN AN EXPANDING QGP}

Our treatment of a fast quark or gluon in a QGP medium has already been discussed in Ref. [15,21]. The behavior is governed by coupled Fokker-Planck equations and rate equations

$$
\begin{aligned}
& \left.\frac{d\left\langle p_{T}^{a}\right\rangle}{d \tau}=-\left\langle\gamma_{a}\left(p_{T}, T\right) p_{T}^{a}\right\rangle \approx-\gamma_{a}\left(\left\langle p_{T}^{a}\right\rangle, T\right)\right)\left\langle p_{T}^{a}\right\rangle, \\
& \frac{d N^{a}}{d \tau}=-\sum_{b} \Gamma^{a \rightarrow b}\left(p_{T}, T\right) N^{a}+\sum_{c} \Gamma^{c \rightarrow a}\left(p_{T}, T\right) N^{c},
\end{aligned}
$$

with letters $a, b$, and $c$ denoting quark, antiquark, gluon, or photon. We have dropped the diffusion term and also neglected the dispersion of jet momentum, i.e., $\left\langle p_{T}^{2}\right\rangle=\left\langle p_{T}\right\rangle^{2}$, because we want to focus on high-momentum jets. The drag coefficient $\gamma_{a}$ is obtained from Ref. [21], including all elastic processes between quarks and gluons. To mimic the contributions from higher-order radiative processes, we introduce an enhancement factor $K$. The conversion widths $\Gamma$ for quark to gluon and vice versa [channels (i) and (iv)] are extracted from Ref. [21] 
as well, multiplied by the same enhancement factor $K$ to account for higher-order corrections. The widths for the quark to photon processes (ii) and (v) at leading order are obtained from Ref. [27].

For a quark or gluon jet moving through the QGP, the rate of change for its mean transverse momentum $\left\langle p_{T}\right\rangle$ can be obtained from the Fokker-Planck equation in Eq. (6). A quark or gluon can be converted to a gluon, quark, or photon with a rate given by the corresponding collisional width. These effects are modeled by introducing a large number of test quark and gluon jets that are distributed in the transverse plane according to the underlying binary nucleon-nucleon collisions. Their transverse-momentum distribution is taken to be uniform in azimuthal angle $\phi$. A test jet of a given kind with certain transverse momentum is assigned a probability that is proportional to the corresponding jet-momentum spectrum with the proportionality constant determined by requiring that the sum of the probabilities for all test jets of this kind is equal to their total number. More details can be found in Ref. [21].

Let us quickly discuss the possible lessons that can be learned by looking at a simplified situation. We assume a rare jet flavor that can be produced from a plentiful reservoir of a second jet flavor via a single channel with width $\Gamma \sim 1 / \lambda$. As long as the first flavor stays rare the back-reaction is negligible. A real world example close to this hypothetical situation could be photons produced from light quark jets at RHIC. Then the rate to produce an additional excess of the rare flavor is

$$
\frac{d N_{\text {rare }}}{d \tau} \approx \frac{1}{\lambda} N_{\text {res }}
$$

Let us further assume that $N_{\text {res }}$ is roughly constant during the lifetime of jets in the system (as for the real world example: up to $30 \%$ of quark jets at RHIC leak to gluon jets [21]). Then we arrive at a simple formula connecting yields and the mean free path for the single channel.

$$
\frac{\lambda}{L} \approx \frac{N_{\text {res }}}{N_{\text {rare,excess }}} .
$$

Obviously, realistic systems are more complicated with multiple channels, changing rates $\Gamma$ due to the temperature evolution of the system, and a whole range of possible path lengths $L$. Therefore, in the following section we will apply our test particle Monte Carlo simulation to study the propagation of jets in an expanding QGP fireball.

\section{RESULTS FOR SPECTRA, $R_{A A}$, AND $v_{2}$ AT RHIC}

In $p+p$ collisions, direct photons come from initial hard collisions and vacuum bremsstrahlung off jets. Bremsstrahlung photons can be obtained by folding the QCD with photon fragmentation functions $D_{\gamma /(q, g)}\left(z, Q^{2}\right)$ from Ref. [39]. The spectra of quark and gluon jets are taken from Ref. [26], which fit both pion and proton spectra measured in $p+p$ collisions from STAR, whereas the prompt initial photons are calculated at leading order according to Ref. [39] with a $K$ factor of 1.5. The resulting $p_{T}$ spectrum of photons in $p+p$ collisions at $\sqrt{s_{N N}}=200 \mathrm{GeV}$ is shown in Fig. 1, and it is found to be in good agreement with data from PHENIX [40] at transverse

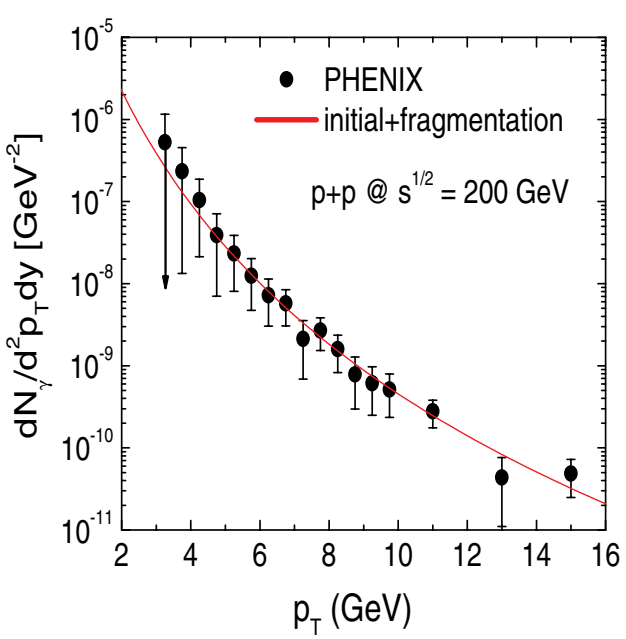

FIG. 1. (Color online) Photon spectrum in $p+p$ collisions at $\sqrt{s_{N N}}=200 \mathrm{GeV}$ as function of transverse momentum. Data are taken from the PHENIX Collaboration [40].

momenta above $4 \mathrm{GeV} / c$. This provides a well-controlled baseline for calculations of the nuclear modification factor $R_{A A}^{\gamma}$.

The yield of additional direct photons in $\mathrm{Au}+\mathrm{Au}$ collisions from jets in the medium is computed by applying the test particle Monte Carlo method discussed above. For the dynamics of the fireball in central collisions we assume that it evolves boost invariantly in the longitudinal direction, but with an accelerated transverse expansion. The parameters for the fireball formed in $\mathrm{Au}+\mathrm{Au}$ collisions at $\sqrt{s_{N N}}=200 \mathrm{GeV}$ are taken from Refs. [15,21,41], where one of us studied energy loss of heavy quark and the $p / \pi^{+}$and $\bar{p} / \pi^{-}$ratios due to jet flavor conversions, as well as production of pentaquark baryons. The volume expands in the proper time $\tau$ according to $V(\tau)=\pi R(\tau)^{2} \tau$, where $R(\tau)=R_{0}+a\left(\tau-\tau_{0}\right)^{2} / 2$ is the transverse radius with an initial value $R_{0}=7 \mathrm{fm} / c, \tau_{0}=$ $0.6 \mathrm{fm} / c$ is the QGP formation time, and $a=0.1 c^{2} / \mathrm{fm}$ is the transverse acceleration. Starting with an initial temperature $T_{0}=350 \mathrm{MeV}$, the time dependence of the temperature is obtained from entropy conservation, leading to the critical temperature $T_{c}=175 \mathrm{MeV}$ at proper time $\tau_{c}=5.0 \mathrm{fm} / c$.

We show all results with two extreme assumptions for the $K$ factor of the jet-plasma interactions. We explore the scenario $K=0$, which simply switches off all flavor conversions. We also show computations with $K=4$. This rather large value is likely to establish an upper bound for the effect, but it is also compatible with the previous work in Ref. [21]. Of course, taking such a large value would imply that a leading-order perturbative treatment is not sufficient to begin with. Note that we keep a separate value of $K=1$ for conversions with a photon in the final state. We have fixed this value with the available data on direct photons.

The resulting spectra of direct photons in $\mathrm{Au}+\mathrm{Au}$ collisions at $\sqrt{s_{N N}}=200 \mathrm{GeV}$ are plotted in Fig. 2 as functions of transverse momentum. The left panel shows the scenario with jet flavor and jet-photon conversions included, whereas the right panel neglects conversions. The results show that direct photons from jet-photon conversions (dashed line in the left 

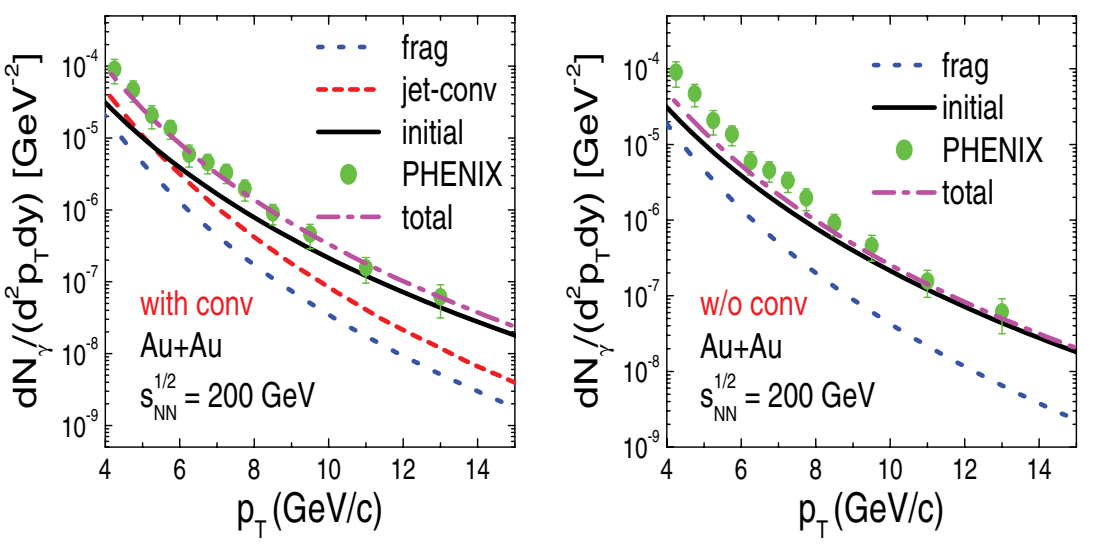

FIG. 2. (Color online) Photon spectra in central $\mathrm{Au}+\mathrm{Au}$ collisions at $\sqrt{s_{N N}}=200 \mathrm{GeV}$ as functions of transverse momentum with (left panel) and without conversions (right panel). Direct photons are from initial hard collisions (solid line), final state jet fragmentation (dotted line), and jet-photon conversions (dashed line). Data are taken from PHENIX [42]. panel) make a sizable contribution below $p_{T}=6 \mathrm{GeV} / \mathrm{c}$ at RHIC, in accordance with previous results [27]. Jet flavor conversions reduce the number of high-momentum quark jets by $30 \%$ [21]. Therefore, conversions lead to a decreased production of direct photons from final-state jet fragmentation (dotted lines in both left panel and right panel) by roughly the same amount.

The nuclear modification factor $R_{A A}^{\gamma}$ for the total direct photon spectrum is shown in the left panel of Fig. 3 (the number of binary collisions is taken to be $\approx 960$ for the most cental bin). The result with all conversion turned off $(K=0)$ is plotted with a dotted line, whereas that with jet conversions included is indicated by the solid line. We find a noticeable difference between both cases. In our calculation the result with conversions is more compatible with the data from PHENIX. Note that the difference between both scenarios decreases with increasing transverse momentum because prompt hard photons from initial scatterings, unchanged by the nuclear environment, dominate at higher $p_{T}$.

As we discussed above, it might be interesting to look at double ratios of nuclear modification factors to extract jet conversion effects. In the middle panel of Fig. 3 we present our expectation for $r_{\gamma / \pi^{+}}=R_{A A}^{\gamma} / R_{A A}^{\pi^{+}}$at intermediate and high $p_{T}$. Note that no recombination effects are taken into account in this study [43], so that deviations in quantities involving hadrons can be expected below $p_{T} \approx 6 \mathrm{GeV} / c$. We find that conversion processes lead to a large signal in $r_{\gamma / \pi^{+}}$. Our results with and without conversion differ by a factor 2 . We also show the double ratio $r_{p / \pi^{+}}=R_{A A}^{p} / R_{A A}^{\pi^{+}}$in the right panel of Fig. 3. Again, we find a large separation between the extreme cases with and without conversions, opening the door for a possible measurement at RHIC.

An azimuthal anisotropy is believed to be generated in a partonic stage in semicentral and peripheral heavy-ion collisions when the fireball has the maximum geometric asymmetry. This anisotropy is studied in terms of Fourier coefficients $v_{2}$ defined from particle yield $d N /\left(p_{T} d p_{T} d \phi\right)$ as

$$
\frac{d N}{p_{T} d p_{T} d \phi}=\frac{d N}{2 \pi p_{T} d p_{T}}\left[1+2 v_{2}\left(p_{T}\right) \cos (2 \phi)+\mathcal{O}(4 \phi)\right]
$$

where the angle $\phi$ is defined with respect to the reaction plane. The coefficient $v_{2}$ can be extracted from the transverse momentum $p_{T}=\left(p_{x}, p_{y}\right)$ of measured particles via

$$
v_{2}=\left\langle\frac{p_{x}^{2}-p_{y}^{2}}{p_{x}^{2}+p_{y}^{2}}\right\rangle
$$
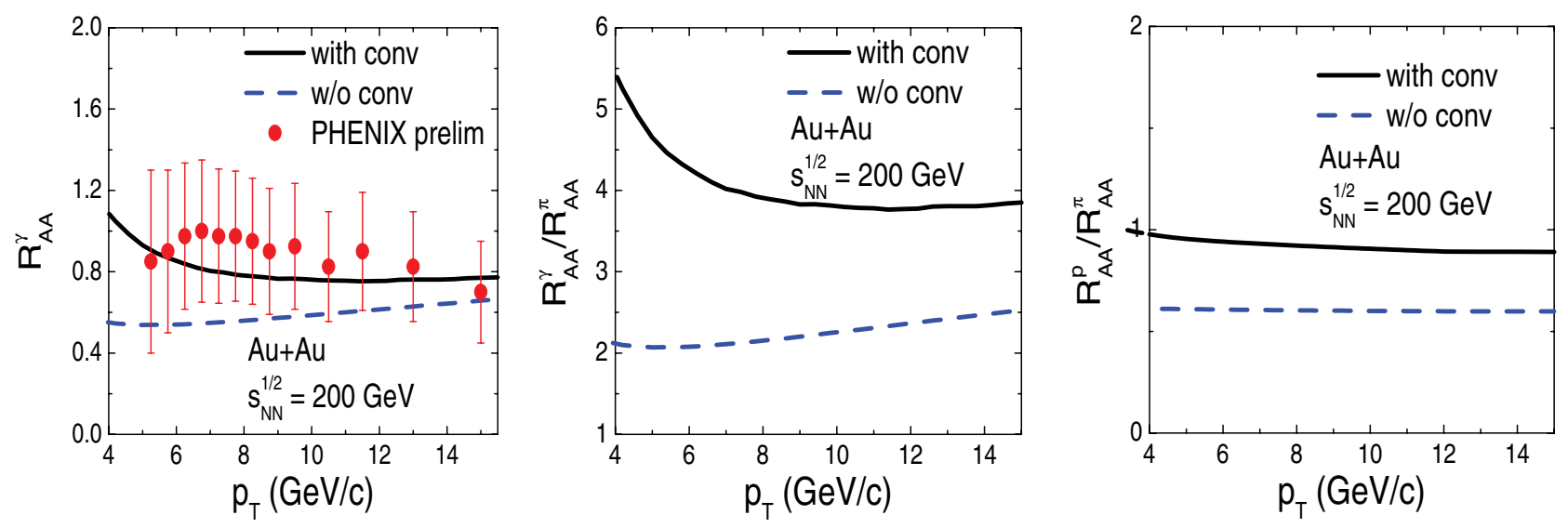

FIG. 3. (Color online) Nuclear modification factor $R_{A A}^{\gamma}$ of direct photons (left panel) and the double ratios of $r_{\gamma / \pi^{+}}=R_{A A}^{\gamma} / R_{A A}^{\pi^{+}}$(middle panel) and $r_{p / \pi^{+}}=R_{A A}^{p} / R_{A A}^{\pi^{+}}$in central Au+Au collisions at $\sqrt{s_{N N}}=200 \mathrm{GeV}$ as functions of transverse momentum $p_{T}$. Results are with (solid line) or without (dotted line) conversions. 

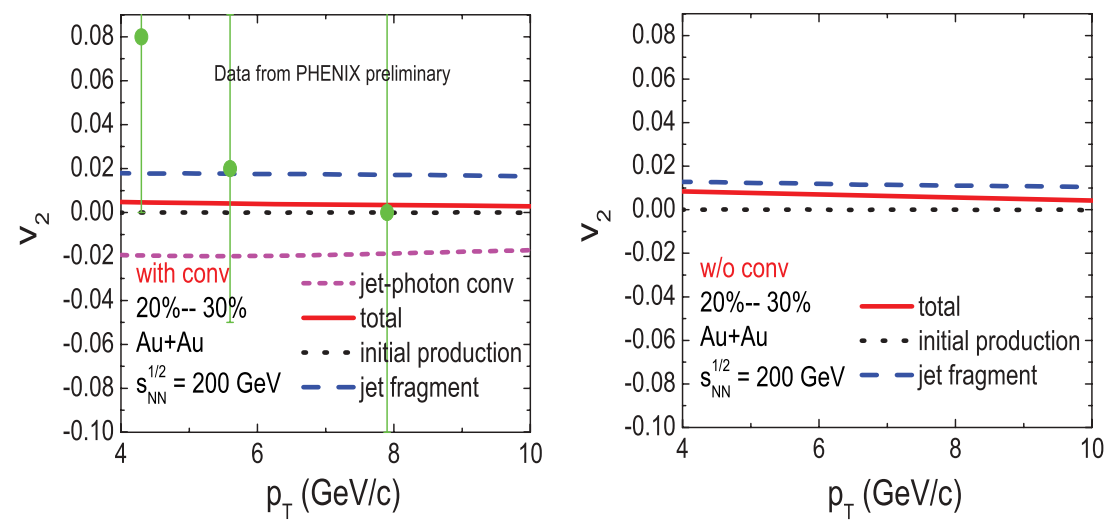

FIG. 4. (Color online) Elliptic flow $v_{2}^{\gamma}$ of direct photons in peripheral $\mathrm{Au}+\mathrm{Au}$ collisions with centrality of $20-30 \%$ at $\sqrt{s_{N N}}=200 \mathrm{GeV}$ as functions of transverse momentum for two different scenarios of with (left panel) or without conversions (right panel), respectively. Data are taken from the PHENIX Collaboration [37]. where the direction of the $x$ axis is in the reaction plane with $\phi=0$ and the direction of the $y$ axis is out of the reaction plane. Here $\langle\cdots\rangle$ means averaging over all measured particles for a given species.

The parameters of the expanding fireball formed in peripheral collisions are extracted from information about centrality, number of participants, and number of binary collisions in $\mathrm{Au}+\mathrm{Au}$ collisions at $\sqrt{s_{N N}}=200 \mathrm{GeV}$ given in Ref. [44]. For example, in collisions in the centrality bin $20-30 \%$, the fireball forms an almond-shaped cylinder with the initial length of the long transverse axis being $l_{L}^{0}=6.1 \mathrm{fm}$ and that of the short transverse axis being $l_{S}^{0}=3.5 \mathrm{fm}$. The acceleration in the direction of the long axis is $a_{L}=0.1 c^{2} / \mathrm{fm}$ and that in the direction of the short axis is $a_{S}=l_{L} / l_{S} a_{L}$. We also assume the fireball is boost invariant and the temperature dependence is obtained from entropy conservation with an initial temperature $T_{0}=340 \mathrm{MeV}$ at proper time $\tau_{0}=0.6 \mathrm{fm} / c$ and critical temperature $T_{c}=175 \mathrm{MeV}$.

The obtained elliptic flow $v_{2}^{\gamma}$ of direct photons is shown in Fig. 4 as a function of $p_{T}$. We plot the results with conversions in the left panel, whereas those in the right panel have $K=0$. The elliptic flow of photons from jet-photon conversions is negative with a magnitude of about $2 \%$, because the production of direct photons in the direction out of the reaction plane is favored by the longer propagation path of jets through the medium [35]. This makes it more likely that a rare jet-photon conversion occurs. $v_{2}^{\gamma}$ of photons from final-state jet fragmentation is positive, following the pattern of $v_{2}$ for hadrons at large $p_{T}$. Adding all contributions together leads to a positive total elliptic flow $v_{2}^{\gamma}$ of direct photons. When jet conversions are switched off, the elliptic flow $v_{2}^{\gamma}$ of direct photons from final-state jet fragmentation decreases because of the larger number of quark jets leaving the fireball. The effects of missing negative conversion $v_{2}$ and increased positive bremsstrahlung $v_{2}$ roughly cancel. Thus we find that the total elliptic flow $v_{2}^{\gamma}$ of direct photons is roughly the same in cases whether or not jet conversions are present. Preliminary data from PHENIX [37] are shown in the left panel. Both cases are compatible with the data within the large experimental uncertainties.

To make this study more complete, it is interesting to explore the centrality dependence of the novel double ratio $R_{A A}^{\gamma} / R_{A A}^{\pi}$ and elliptic flow of direct photons at RHIC. When the centrality of the collisions increases, the resulting decreased average path length will lead to reduction of both energy loss and jet conversion rates. Hence we expect that the difference between the scenarios with and without jet conversions for the double ratio $R_{A A}^{\gamma} / R_{A A}^{\pi}$ will shrink with increasing centrality. For the elliptic flow of direct photons, the centrality dependence is not so straightforward to estimate because changes in energy loss and jet conversion rates try to sway $v_{2}$ in different directions. We calculate the elliptic flow of direct photons produced in $\mathrm{Au}+\mathrm{Au}$ collisions at $\sqrt{s_{N N}}=$ $200 \mathrm{GeV}$ in the more peripheral centrality bins of $30-40 \%$ and $40-50 \%$, respectively, and show the results in Fig. 5. The resulting total elliptic flow of direct photons is just as small as for the $20-30 \%$ centrality bin. Thus direct photon $v_{2}$ is not sensitive to the impact parameter in $\mathrm{Au}+\mathrm{Au}$ collisions due to the cancellation between jet conversions and jet energy loss.

Let us now proceed to discuss the impact of conversions on strange hadrons, in particular kaons. In Ref. [21] only $u$ and $d$ quarks had been included alongside gluons, and the resulting $p / \pi^{+}$and $\bar{p} / \pi^{-}$ratios reproduce the data from STAR [24]. We neglect the relatively small current mass of the strange quark and replace it solely with the thermal mass $\sim g T$ in the medium when calculating its drag coefficient and conversion width. This might slightly overestimate conversion rates to strange quarks, but it simplifies the treatment [21].

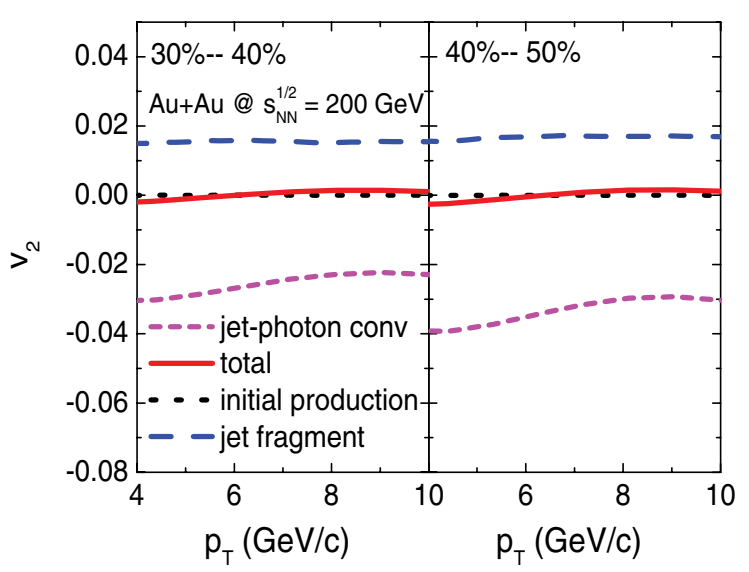

FIG. 5. (Color online) Elliptic flow $v_{2}^{\gamma}$ of direct photons in peripheral $\mathrm{Au}+\mathrm{Au}$ collisions with centrality of $30-40 \%$ (left) and $40-50 \%$ (right) at $\sqrt{s_{N N}}=200 \mathrm{GeV}$ as a function of transverse momentum. 

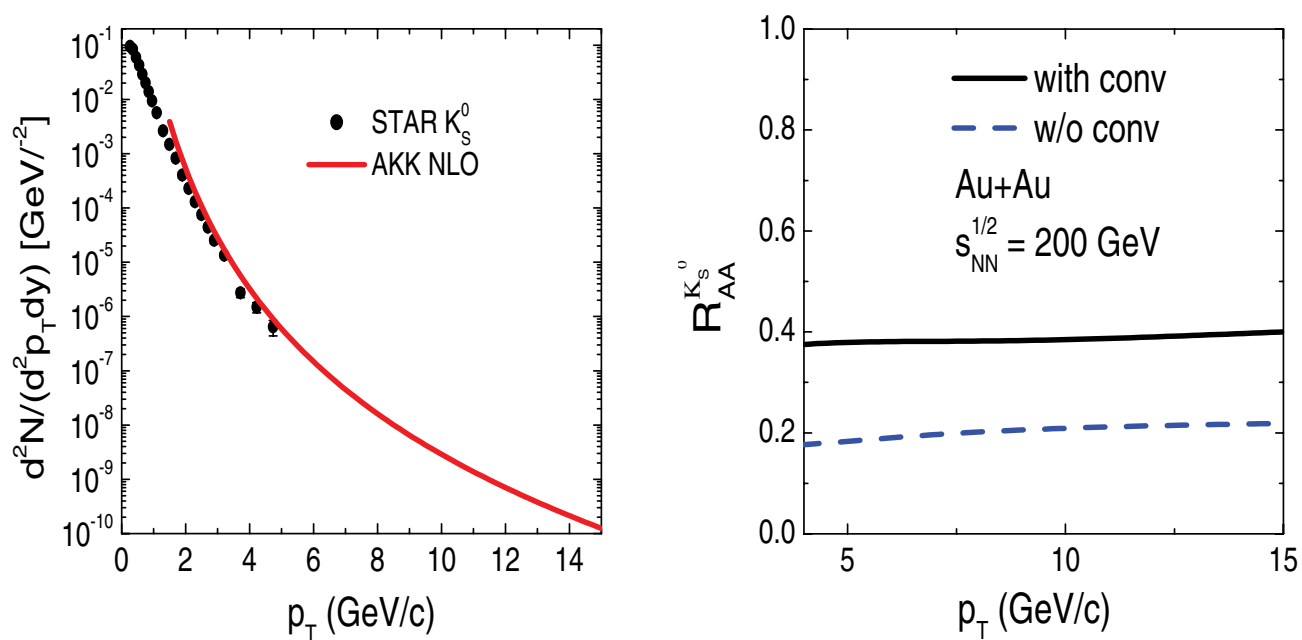

FIG. 6. (Color online) $K_{S}^{0}$ (left panel) spectra from quark and gluon jet fragmentation via AKK fragmentation function [22] in $p+p$ collisions at $\sqrt{s_{N N}}=200 \mathrm{GeV}$. Data are from the STAR Collaboration [45]. Nuclear modification factor $R_{A A}$ (right panel) for $K_{S}^{0}$ in Au+Au collisions at same center-of-mass energy as a function of transverse momentum.

In the left panel of Fig. 6 we show the spectrum of $K_{s}^{0}$ in $p+p$ collisions calculated as a baseline. On the right-hand side we plot the nuclear modification factor $R_{A A}^{K}$ of the $K_{S}^{0}$ as a function of transverse momentum $p_{T}$. We observe that conversions greatly enhance the yield of $K_{S}^{0}$ in nuclear collisions. In fact, conversions could lead to a $R_{A A}$ that is up to a factor 2 larger at high $p_{T}$ than that for pions or protons. Note again that a recombination contribution is not included here, so caution has to be exercised at lower $p_{T}$. We predict that the measurement of the nuclear modification factor of kaons and $\Lambda \mathrm{s}$ at high $p_{T}$ will provide a unique signal for jet conversions in the QGP formed at RHIC.

\section{A LOOK AT LHC}

At Large Hadron Collider (LHC) energies jet physics are expected to play an even bigger role than at RHIC. Using the procedures developed previously we compute the expected nuclear modification factors $R_{A A}^{\gamma}$ and $R_{A A}^{K}$ and the elliptic flow $v_{2}^{\gamma}$ for direct photons as well as various double ratios in $\mathrm{Pb}+\mathrm{Pb}$ collisions at $\sqrt{s_{N N}}=5.5 \mathrm{TeV}$. We want to gauge the effect of jet conversions on high- $p_{T}$ measurements at LHC.

We use jet spectra at $5.5 \mathrm{TeV}$ by multiplying those obtained from PYTHIA in $p+p$ collision at the same energy by the number of binary collisions [26] $\left(\left\langle N_{\text {coll }}\right\rangle \approx 1700\right.$ [46]). We also assume that the fireball produced in central collisions at LHC is cylindrical and Bjorken boost invariant. The parameters of the fireball are taken from Ref. [47], where thermal charm production at LHC was studied. Specifically, we choose initial proper time $\tau_{0}=0.2 \mathrm{fm} / c$ for the formation of the equilibrated QGP with initial temperature $T_{0}=700 \mathrm{MeV}$ and flow acceleration $a=0.1 c^{2} / \mathrm{fm}$. This yields reasonable final transverse flow velocities with initial radius $R_{0}=7.0 \mathrm{fm}$. Fixing the parameters at the critical temperature $T_{c}=$ $175 \mathrm{MeV}$ via entropy conservation we obtain $\tau_{c}=6.3 \mathrm{fm} / c$.
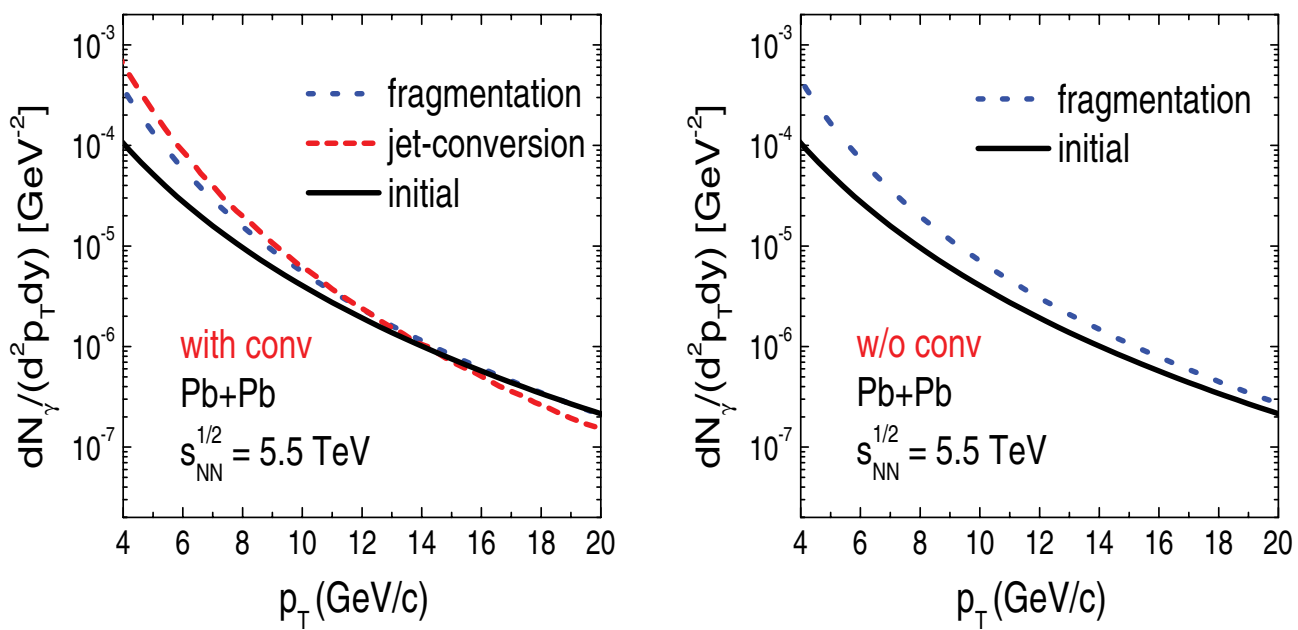

FIG. 7. (Color online) Photon spectra in central $\mathrm{Pb}+\mathrm{Pb}$ collisions at $\sqrt{s_{N N}}=5.5 \mathrm{TeV}$ as functions of transverse momentum $p_{T}$ with (left panel) or without conversions (right panel). 

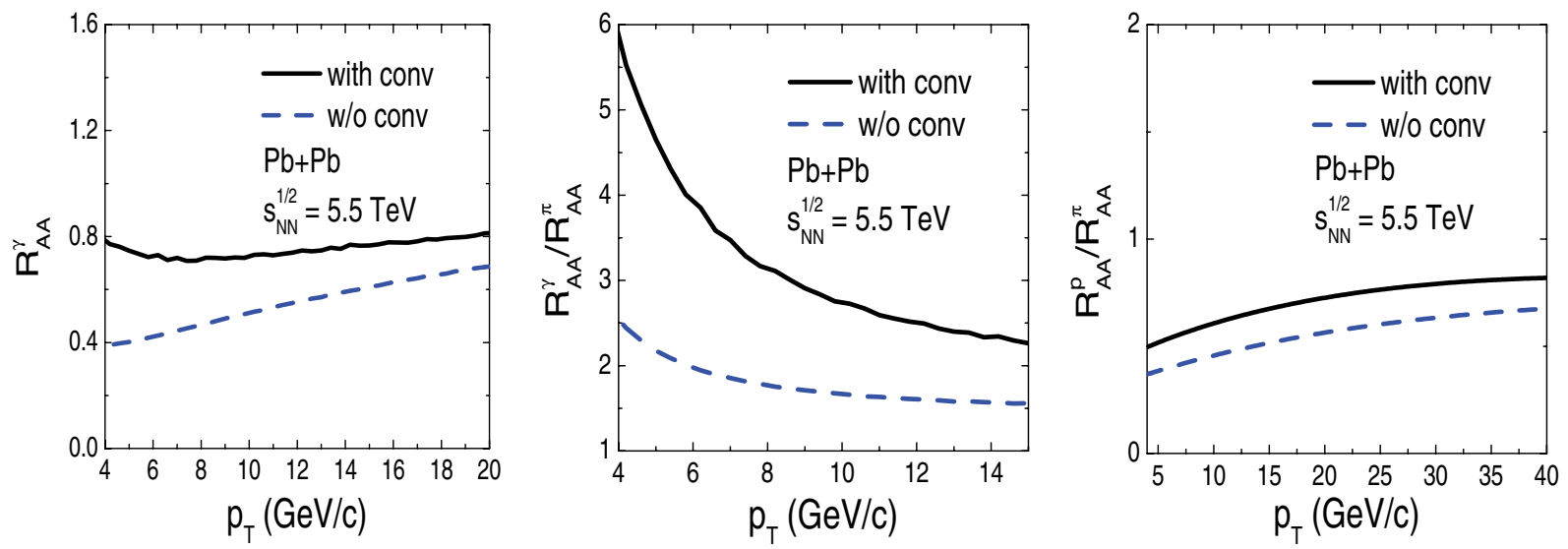

FIG. 8. (Color online) Same quantities as those in Fig. 3 in central $\mathrm{Pb}+\mathrm{Pb}$ collisions at $\sqrt{s_{N N}}=5.5 \mathrm{TeV}$ as functions of transverse momentum with or without conversions.

We apply the same test particle Monte Carlo method as above with the same set of $K$ factors.

Our results for direct photons are shown in Fig. 7 for $K=4$ (1 for photons) (left panel) and $K=0$ (right panel), respectively. Direct photons from jet-photon conversions and jet fragmentation dominate below $p_{T}=16 \mathrm{GeV} / c$ when conversions are switched on, with photons from conversion being the largest source below $10 \mathrm{GeV} / c$. Without jet conversions the direct photons from jet fragmentation are always brighter than those from initial hard collisions up to $20 \mathrm{GeV} / c$, because of the reduced conversion of quarks.

It is straightforward to calculate the nuclear modification factor $R_{A A}^{\gamma}$, which is shown in the left panel of Fig. 8. The difference between both scenarios can be as large as a factor of 2 at $p_{T}=4 \mathrm{GeV} / c$ and decreases to a factor of about 1.25 at $20 \mathrm{GeV} / c$. We also observe a large increase in the double ratio $r_{\gamma / \pi^{+}}=R_{A A}^{\gamma} / R_{A A}^{\pi^{+}}$if conversions are included, just as seen for RHIC. For comparison, we also show the result of the double ratio $r_{p / \pi^{+}}$at LHC.

Because we have seen that direct photons at LHC are mostly from jet-photon conversions and final-state jet fragmentation, they are strongly influenced by jet conversions. Photon elliptic flow $v_{2}^{\gamma}$ might reveal those large effects. The parameters of the fireball formed in peripheral collisions at LHC are extracted from information about centrality, number of participants, and number of binary collisions in $\mathrm{Pb}+\mathrm{Pb}$ collisions at $\sqrt{s_{N N}}=5.5 \mathrm{TeV}$ [46]. For simplicity, in collisions at LHC within the centrality bin $20-30 \%$, we use the same geometric parameters (the length of the long and short axes) for the initial fireball as at RHIC, but we set the initial temperature and proper time of QGP formation to $T_{0}=700 \mathrm{MeV}$ and $\tau_{0}=0.2 \mathrm{fm} / c$, respectively, consistent with central collisions. The time dependence of the temperature is also fixed via entropy conservation.

In Fig. 9 we show the resulting elliptic flow $v_{2}^{\gamma}$ of direct photons in $\mathrm{Pb}+\mathrm{Pb}$ collisions at $\sqrt{s_{A A}}=5.5 \mathrm{TeV}$ with and without conversions. The elliptic flow from photon fragmentation off jets is positive with a magnitude of $2 \%$ in the case of jet conversions, but it is reduced by a factor of 2 in the case of missing jet conversions due to the reduced quenching for quark. The resulting total elliptic flow of direct photons without jet conversions differs only slightly from that of jet fragmentation alone, because direct prompt photons from the initial state are subdominant below $20 \mathrm{GeV} / c$. With jet conversions included, we again observe a cancellation between the negative anisotropy from jet-photon conversions and the increase in jet fragmentation with positive anisotropy, leading to a total elliptic flow $v_{2}^{\gamma}$ of almost the same magnitude as that without jet conversions. Together with our previous result at RHIC energies this might indicate that photon elliptic flow,
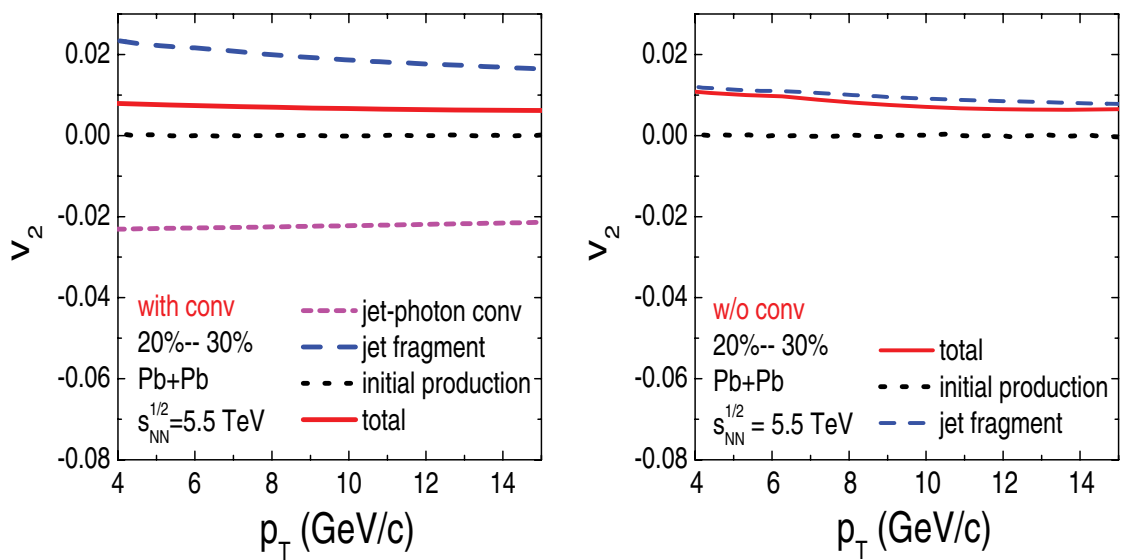

FIG. 9. (Color online) Elliptic flow $v_{2}^{\gamma}$ of direct photons in peripheral $\mathrm{Pb}+\mathrm{Pb}$ collisions with centrality of $20-30 \%$ at $\sqrt{s_{N N}}=$ $5.5 \mathrm{TeV}$ as functions of transverse momentum with (left panel) or without conversions (right panel). 


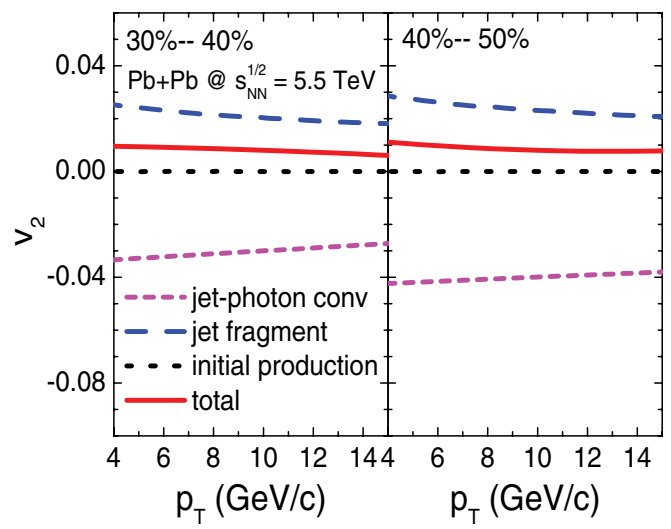

FIG. 10. (Color online) Elliptic flow $v_{2}^{\gamma}$ of direct photons in peripheral $\mathrm{Pb}+\mathrm{Pb}$ collisions with centrality of $30-40 \%$ (left) and $40-50 \%$ (right) at $\sqrt{s_{N N}}=5.5 \mathrm{TeV}$ as functions of transverse momentum.

despite its early promise, might need very large experimental precision to be used as a probe. The situation might be much improved, however, if measurements with additional isolation cuts could be made [35].

We also predict the centrality dependence of the elliptic flow for direct photons in $\mathrm{Pb}+\mathrm{Pb}$ collisions at $\sqrt{s_{N N}}=$ $5.5 \mathrm{TeV}$ and show the results in Fig. 10. The numerical results confirm once more that the total elliptic flow of direct photons at high transverse momentum varies only weakly with the centrality of the collision.

In Fig. 11 we show $R_{A A}^{K}$ of $K_{S}^{0}$ in central collisions at LHC. We observe that jet conversions have only a small impact on $R_{A A}^{K}$. This had to be expected, because initial jet production is gluon dominated and strange quark jets are not very much suppressed to begin with, very unlike the situation found at RHIC. The impact of jet conversions on the $R_{A A}$ of kaons is similar to the effect on pions at LHC [20]. We conclude that strange hadrons might no longer be a good probe of jet conversions at LHC energies.

\section{SUMMARY AND DISCUSSION}

We studied the impact of flavor conversions of jets on observables in heavy-ion collisions. We argued that the relaxation of jet flavors toward chemical equilibrium can be an independent measure for the strength of the jet-medium coupling. We presented a computation of several observables that are sensitive to conversion processes at RHIC and LHC

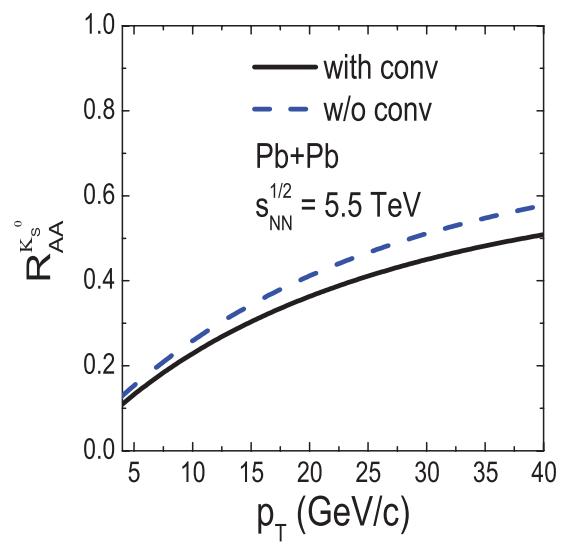

FIG. 11. (Color online) Nuclear modification factor $R_{A A}$ for $K_{S}^{0}$ in $\mathrm{Pb}+\mathrm{Pb}$ collisions at $\sqrt{s_{N N}}=5.5 \mathrm{TeV}$ as function of transverse momentum.

using a Fokker-Planck approach and coupled rate equations for conversions. Rates were based on leading-order cross sections with a $K$ factor.

We find a large change in the nuclear modification factor $R_{A A}^{\gamma}$ when jet conversions are switched on, whereas the effects on photonic elliptic flow $v_{2}^{\gamma}$ tend to cancel. We find our results to be consistent with the available experimental data from PHENIX [40]. We also calculated the impact of jet conversions on the double ratios of nuclear modification factor $r_{\gamma / \pi^{+}}=R_{A A}^{\gamma} / R_{A A}^{\pi^{+}}$and $r_{p / \pi^{+}}=R_{A A}^{p} / R_{A A}^{\pi^{+}}$and find that these quantities are quite sensitive to the presence of jet conversions. The difference can be as large as a factor of 2 . At the same time such double ratios might be extracted from experimental data with rather small systematic error bars. Large $K$ factors in our leading-order treatment would hint at nonperturbative mechanisms, or to the presence of additional effects, like the influence of increased multiplicities in modified jets [19]. We also advocate the measurement of strange hadrons at high $p_{T}$ as a possible signature for the jet-medium coupling. The low initial strange quark content of jets at RHIC makes kaons and other strange hadrons a a very sensitive probe for the coupling to the medium.

\section{ACKNOWLEDGMENTS}

We thank Che-Ming Ko for useful discussions and suggestions. This work was supported by RIKEN/BNL, DOE grant DE-AC02-98CH10886, and the Texas A\&M College of Science.
[1] J. Adams et al. (STAR Collaboration), Nucl. Phys. A757, 102 (2005); K. Adcox et al. (PHENIX Collaboration), ibid. A757, 184 (2005).

[2] A. Adcox et al. (PHENIX Collaboration), Phys. Rev. Lett. 88, 022301 (2002).

[3] C. Adler et al. (STAR Collaboration), Phys. Rev. Lett. 89, 202301 (2002); 90, 082302 (2002).

[4] R. Baier, Y. L. Dokshitzer, A. H. Mueller, S. Peigne, and D. Schiff, Nucl. Phys. B483, 291 (1997); B484, 265 (1997).
[5] B. G. Zakharov, JETP Lett. 63, 952 (1996).

[6] X. N. Wang, Phys. Lett. B579, 299 (2004).

[7] M. Gyulassy, P. Lévai, and I. Vitev, Phys. Rev. Lett. 85, 5535 (2000); Nucl. Phys. B594, 371 (2001).

[8] U. A. Wiedemann, Nucl. Phys. B588, 303 (2000).

[9] P. Arnold, G. D. Moore, and L. G. Yaffe, J. High Energy Phys. 06 (2002) 30; S. Jeon and G. D. Moore, Phys. Rev. C 71, 034901 (2005).

[10] B. Sahlmueller, J. Phys. G 34, S969 (2007). 
[11] E. V. Shuryak, Phys. Rev. C 66, 027902 (2002).

[12] B. I. Abelev et al. (STAR Collaboration), Phys. Rev. Lett. 98, 192301 (2007).

[13] M. G. Mustafa, Phys. Rev. C 72, 014905 (2005); M. G. Mustafa and M. H. Thoma, Acta Phys. Hung. A 22, 93 (2005).

[14] S. Wicks, W. Horowitz, M. Djordjevic, and M. Gyulassy, Nucl. Phys. A784, 426 (2007).

[15] W. Liu and C. M. Ko, preprint nucl-th/0603004; W. Liu and C. M. Ko, J. Phys. G. 34, s775 (2007); C. M. Ko and W. Liu, Nucl. Phys. A783, 233 (2007).

[16] H. van Hees and R. Rapp, Phys. Rev. C 71, 034907 (2005).

[17] C. P. Herzog, A. Karch, P. Kovtun, C. Kozcaz, and L. G. Yaffe, J. High Energy Phys. 07 (2006) 13; H. Liu, K. Rajagopal, and U. A. Wiedemann, Phys. Rev. Lett. 97, 182301 (2006).

[18] A. Schäfer, X. N. Wang, and B. W. Zhang, Nucl. Phys. A793, 128 (2007).

[19] S. Sapeta and U. A. Wiedemann, preprint, arXiv:0707.3494 [hep-ph].

[20] W. Liu and R. J. Fries, arXiv: 0801.0453 [nucl-th].

[21] W. Liu, C. M. Ko, and B. W. Zhang, Phys. Rev. C 75, 051901(R) (2007); W. Liu, C. M. Ko, and B. W. Zhang, Int. J. Mod. Phys. E 16, 1930 (2007); C. M. Ko, W. Liu, and B. W. Zhang, Few-Body Syst. 41, 63 (2007).

[22] S. Albino, B. A. Kniehl, and G. Kramer, Nucl. Phys. B725, 181 (2005).

[23] J. Adams et al. (STAR Collaboration), Phys. Lett. B637, 161 (2006).

[24] J. Adams et al. (STAR Collaboration), Phys. Rev. Lett. 97, 152301 (2006).

[25] X. N. Wang, Phys. Rev. C 58, 2321 (1998).

[26] W. Liu, C. M. Ko, and B. W. Zhang (in preparation).

[27] R. J. Fries, B. Muller, and D. K. Srivastava, Phys. Rev. Lett. 90, 132301 (2003).

[28] R. J. Fries, B. Muller, and D. K. Srivastava, Phys. Rev. C 72, 041902(R) (2005).
[29] C. Gale, T. C. Awes, R. J. Fries, and D. K. Srivastava, J. Phys. G 30, S1013 (2004).

[30] S. Turbide, C. Gale, S. Jeon, and G. D. Moore, Phys. Rev. C 72, 014906 (2005).

[31] S. Turbide, C. Gale, E. Frodermann, and U. Heinz, arXiv:0712.0732 [hep-ph].

[32] D. K. Srivastava, C. Gale, and R. J. Fries, Phys. Rev. C 67, 034903 (2003); S. Turbide, C. Gale, D. K. Srivastava, and R. J. Fries, ibid. 74, 014903 (2006).

[33] S. S. Adler et al. (PHENIX Collaboration), Phys. Rev. Lett. 94, 232301 (2005).

[34] T. Isobe (PHENIX Collaboration), J. Phys. G 34, S1015 (2007).

[35] S. Turbide, C. Gale, and R. J. Fries, Phys. Rev. Lett. 96, 032303 (2006).

[36] S. S. Adler et al. (PHENIX Collaboration), Phys. Rev. Lett. 96, 032302 (2006).

[37] V. S. Pantuev (PHENIX Collaboration), J. Phys. G 34, S805 (2007).

[38] H. L. Lai et al. (CTEQ Collaboration), Eur. Phys. J. C 12, 375 (2000).

[39] J. F. Owens, Rev. Mod. Phys. 59, 465 (1987).

[40] S. S. Adler et al. (PHENIX Collaboration), Phys. Rev. Lett. 98, 012002 (2007).

[41] L. W. Chen, V. Greco, C. M. Ko, S. H. Lee, and W. Liu, Phys. Lett. B601, 34 (2004).

[42] S. S. Adler et al. (PHENIX Collaboration), Phys. Rev. Lett. 94, 232301 (2005).

[43] R. J. Fries, B. Muller, C. Nonaka, and S. A. Bass, Phys. Rev. Lett. 90, 202303 (2003); Phys. Rev. C 68, 044902 (2003).

[44] S. S. Adler et al. (PHENIX Collaboration), Phys. Rev. Lett. 91, 072301 (2003).

[45] B. I. Abelev et al. (STAR Collaboration), Phys. Rev. C 75, 064901 (2007).

[46] D. d'Enterria, preprint, nucl-ex/0302016.

[47] B. W. Zhang, C. M. Ko, and W. Liu, Phys. Rev. C 77, 024901 (2008). 